\title{
An Alternative Approach to Sending All Gallbladders for Histology Following Cholecystectomy?
}

\author{
John-Patrick Devine Byars ${ }^{1 *}$, Kishore Pursnani ${ }^{2}$ \\ ${ }^{1}$ School of Medicine, Manchester University, Manchester, UK \\ ${ }^{2}$ Department of General Surgery, Royal Preston Hospital, Preston, UK \\ Email: *John-patrick.byars@doctors.org.uk
}

Received August 5, 2011; revised September 29, 2011; accepted November 29, 2011

\begin{abstract}
Background: The question of a selective approach to sending all gallbladders for histology following cholecystectomy has been postulated in many journals but as yet no guidelines have been published in light of such concern. This project will attempt to analyse and address the controversy surrounding sending all gallbladders for histology following cholecystectomy. Objective: To ascertain whether there is a feasible and safe alternative to sending all gallbladders to histology following cholecystectomy and to challenge such a hypothesis. Design: Retrospective study. Methods: Analysis of gallbladder pathology reports, patient medical records and computerised test results from cholecystectomies done in the last 5 years within the Lancashire Teaching Hospital Trust. Results: The alternative selective method based on pre-operative investigations and intra-operative macroscopic detail is shown to be significant $(\mathrm{P}<0.05)$. Conclusion: The results from this study show that there is safe and efficient way of selecting which gallbladders are sent for histology based on age, pre-surgical suspicion and intra-operative macroscopic abnormalities.
\end{abstract}

Keywords: Cholecystectomy; Gallbladder; Pathology; Histology; Laparoscopic

\section{Introduction}

Although the fifth most common cancer of the bile tract, primary carcinoma of the gallbladder is uncommon [1]. Coupled to its rarity is the high mortality rate with a 5 year survival rate of less than $5 \%$ and a median survival of only 3 months [2]. In most NHS hospitals all gallbladders are sent for histology following cholecystectomy regardless of the presence of macroscopic abnormalities. This has a direct impact on histopathologists especially in the current era of sub-specialization, increased clinical demand, more detailed analysis of specimens and minimum standards of reporting on difficult cases [3]. Evidence has emerged that suggests early carcinoma of the gallbladder $\left(\mathrm{pT}_{1}\right)$ need not be treated further than the cholecystectomy that was previously performed in order to obtain the tissue sample. In other words, although early macroscopic findings indicative of carcinoma of the gallbladder could theoretically be missed in a selective method, no further treatment is necessary in such early pathogenic stages [4]. The question of a selective approach to sending all gallbladders for histology following cholecystectomy has been postulated in many journals but as yet no guidelines have been published in light of such concern [5-9] This project will attempt to

\footnotetext{
"Corresponding author.
}

analyse and address the controversy surrounding sending all gallbladders for histology following cholecystectomy.

\section{Methods}

Data used in the study was analysed from those patients that had undergone either a laparoscopic or open cholecystectomy between 30th March 2005 and 31st March 2010 in the Lancashire Teaching Hospital Trust. In total there were 2696 gallbladder pathology reports. The histopathological analysis of the gallbladder specimens were conducted by several pathologists all working in the Lancashire Teaching Hospital Trust.

The main outcome of this study was to determine whether there was a viable alternative to sending every single gallbladder for histopathological analysis. The hypothesis was that an alternative method had to be as effective as sending all gallbladders for histology. Therefore in order for the study to be successful, all gallbladder carcinoma patients must be identified pre-operatively. Added to this, there must be a marked reduction in the number of gallbladders sent for histology, represented by those patients that do not have any identifiable risk factors. The number of false negatives should be zero and the number of false positives must be substan- 
tially lower than the total number in the study.

As all patients positive for carcinoma of the gallbladder were female, all subjects used in this analysis were as such female, therefore omitting discrepancy of the results on part of the cohort of patient's sex. In order to allow a fair comparison of the findings that would normally be found in an aging individual, the subjects used as a comparison to the carcinoma patients were also age-matched. Where possible 15 patients were used as a comparison to each gallbladder carcinoma patient. In those cases where there was not 15 patients of the same age and sex, as many similar patients were used that were of the same sex and as close to their age as possible. This brought to a total 89 patients, 7 of which were excluded from the study as a result of their medical records not being retrievable.

For the remaining 82 patients used in this study specific details were documented on a database. This included their age at cholecystectomy, Ultrasound scan (USS) findings and the surgeon's intra-operative macroscopic findings. For those patients that had USS findings indicative or suspicious of gallbladder carcinoma further information was sought from patient notes on whether they had, if any, further radiological investigations preceding surgery and their findings. Information was also collected for those patients that had no investigations preceding surgery but their reasons why were not identified. Lastly there was a column that was highlighted if the patient satisfied the criteria that would result in their specimen being sent for histology.

The criteria for sending the gallbladder for histology was either an USS finding indicative or suspicious of gallbladder carcinoma, other investigations that were indicative or suspicious of gallbladder cancer or red flagged surgeon's intra-operative macroscopic findings.
The criteria for identifying those findings that would be deemed indicative or suspicious for gallbladder cancer on USS were evidenced based on published articles by Jang J. Y. et al. [10] and Levy A. D. et al. [11].

Those findings that were deemed strongly suggestive of carcinoma of the gallbladder on USS were: Irregular margins [11] i.e. irregular mucosal or irregular wall thickening, including mucosal change combined with loss of normal mural layers [10]; Heterogenous echotexture-reflects varying degrees of tumour necrosis [11]; Focal mass [11]; Suspected lymphadenopathy [11]; Echogenic foci and acoustic shadowing associated with tumour, which may be related to co-existing gallstones, gallbladder calcification or tumoural calcification [11]; Porcelain gallbladder [11]; Calcified gallstones [11] and lastly, calcification precipitating in mucus within the neoplastic glandular tissue [11].

Those findings that were deemed suspicious on USS were: Dilated hepatic ducts and biliary obstruction at the level of the porta hepatis [11]; Abnormal collection of gas in right upper quadrant, when tumour has invaded adjacent bowel and fistula has formed [11] and gas in the biliary tree [11].

USS findings were also documented when they represented well-known radiological findings such as: Adenomyoma(tosis)-when intramural cysts of echogenic foci with a ring-down or twinkling artifact were observed [10]; Cholesterolosis-when uniform hyperechogenic mucosal thickening was observed [10]; Cholecystitis-when an intact or disrupted mucosal layer without mucosal thickening was observed with gallbladder wall thickening [10] and lastly, whether or not there was calculi present. Normal gallbladder findings on USS were also documented (Table 1).

Findings that would be highlighted as being high to

Table 1. Summarized USS finding.

\begin{tabular}{|c|c|c|}
\hline $\begin{array}{c}\text { USS findings strongly suggestive of } \\
\text { carcinoma }\end{array}$ & USS findings suggestive of carcinoma & Other USS findings \\
\hline Irregular margins & Gas in the biliary tree & $\begin{array}{l}\text { Adenomyoma(tosis) - when intramural cysts or } \\
\text { echogenic foci within a ring down or twinkling } \\
\text { artifact were observed }\end{array}$ \\
\hline Heterogenous echotexture & Abnormal collection of gas in the RUQ & $\begin{array}{l}\text { Cholesterolosis-when uniform hyperechogenic } \\
\text { mucosal thickening was observed }\end{array}$ \\
\hline Porcelain gallbladder & Biliary obstruction at the level of the porta hepatis & Calculi present \\
\hline Calcified gallstones & & Normal \\
\hline \multicolumn{3}{|l|}{ Focal mass } \\
\hline $\begin{array}{l}\text { Calcification precipitating in mucus within } \\
\text { the neoplastic glandular tissue }\end{array}$ & & \\
\hline
\end{tabular}


moderate risk for carcinoma of the gallbladder intraoperatively was a thick-walled gallbladder, adhesions, a gallbladder that was hard to touch and a gallbladder that was attached to the gallbladder bed. Such findings were collected from the surgeon's post-surgical report present in the patient's medical records. All patients had their gallbladders macroscopically examined intra-operatively by the surgeon during the cholecystectomy surgical procedure as is standard protocol in the Trust.

All information in this study was obtained from pathology reports, patient's medical records and computerised resources to obtain the medical radiological results.

Analysis of the data was conducted using Fisher-exact test with the statistical analysis program Stats Direct. $\mathrm{P}<$ 0.05 was deemed as significant in this study.

\section{Results}

Of the 2696 patients that were included in the study, 662 $(24.5 \%)$ were male and 2034 (75.5\%) were female. The minimum age of patient that underwent a cholecystectomy was 8 years old and the oldest 90 years old. The median age was 51.2 years old. 738 patients $(27 \%)$ were $\leq 40$ years old; 1069 patients $(40 \%)$ were between 41 and 59 years old and 889 patients $(33 \%)$ were $\geq 60$ years old at the time of their cholecystectomy. 7 patients $(0.26 \%)$ were found to have carcinoma of the gallbladder, all of which were female. The ages of the patients that were positive for gallbladder carcinoma ranged from 59 to 90 years old with an average of 75.4 years. 5 out of the 7 gallbladder carcinoma patients $(71.4 \%)$ satisfied the criteria for pre-operative suspicion of carcinoma on the basis of radiological investigation. Of the 5 patients that had pre-surgical suspicion of carcinoma all of them had further investigations which also reinforced suspicion of carcinoma. All 7 patients $(100 \%)$ satisfied the criteria in the study for being referred to histology on suspicion of cancer on the basis of intra-operative macroscopic abnormalities including patient 4 in which an investigative laparotomy led to a biopsy diagnosing the carcinoma (Carcinoma of the gallbladder was suspected during the operation which led to the biopsy taking place). 1 patient $(0.04 \%)$ was diagnosed with non-specific adenocarcinoma, 2 patients $(0.07 \%)$ were diagnosed with poorly differentiated adenocarcinoma and 4 patients $(0.15 \%)$ were diagnosed with moderately differentiated adenocarcinoma of the gallbladder (Table 2).

The study hypothesized whether an alternative to sending all gallbladders for histology could be feasible. By using an age and sex matched selection of patients in this study it would allow us to preliminarily test our alternative system of selecting patients based on radiologycal investigations and intra-operative macroscopic findings. There were in total 82 patients in this study, 7 of which were positive for adenocarcinoma of the gallbladder. 38 patients $(46.3 \%)$ were found to have no suspicious appearances on USS or other radiological investigations suggestive of carcinoma. Coupled to that there were no abnormal appearances of the gallbladder noted during surgery. All 38 patients were found to have benign conditions when correlated with the histopathology diagnosis. 3 patients $(3.7 \%)$ were found to have suspicious findings on radiological investigations but were later reported to have a normal looking gallbladder during surgery. All 3 patients were concluded to have benign conditions when correlated with the histopathology diagnosis. 10 patients $(12.2 \%)$ were found to have suspicious appearances on radiological investigations as well as an abnormal looking gallbladder intra-operatively. 5 of the patients were positive for carcinoma of the gallbladder while the other 5 were diagnosed with benign conditions. 31 patients $(37.8 \%)$ were found to have nonsuspicious looking gallbladders on radiological investigations but satisfied the criteria of an abnormal gallbladder intra-operatively. 2 of the patients in this category were found to have carcinoma of the gallbladder and the remaining 29 patients were diagnosed with benign conditions following their gallbladders being sent for histology. The statistical significance of this data was found to be significant with a p value of 0.013 (Table 3 ).

\section{Discussion}

The prognosis of gallbladder carcinoma is notoriously poor mostly due to the absence of specific symptoms and signs with the majority of patients presenting with advanced disease, [9] with a 5 year survival rate of less than $5 \%$ and a median survival of only 3 months [2]. With the advent of laparoscopic surgery, cholecystectomies have become one of the most common surgical procedures performed. Laparoscopic cholecystectomy appears to have resulted in earlier discovery of gallbladder carcinoma in some patients. The result of this should translate therefore to increased probability of survival [12]. Nevertheless, despite this increase in cholecystectomy frequency, there has been no increase in the incidence of gallbladder carcinoma or dysplasia [13]. The paradox becomes apparent when you become aware of the current guidelines in most NHS hospitals of sending all gallbladders to histology. As such a more effective and selective method is needed, though one that does not compromise patient safety.

The criteria hypothesized in this study would not claim to be $100 \%$ effective in detecting all early carcinomas of the gallbladder. Nevertheless, it could be argued quite convincingly that the criteria need not be. There has emerged strong evidence that substantiates that the management of early carcinoma of the gallbladder (stage $\mathrm{pT}_{1}$ ) 
Table 2. Details of patients with carcinoma of the gallbladder.

\begin{tabular}{cccccccc}
\hline Pt. no. & $\begin{array}{c}\text { Age } \\
\text { (yr) }\end{array}$ & M/F & $\begin{array}{c}\text { Pre-operative } \\
\text { suspicion on USS }\end{array}$ & $\begin{array}{c}\text { Other Ix. } \\
\text { performed } \\
\text { following USS }\end{array}$ & $\begin{array}{c}\text { Suspicious findings } \\
\text { on other Ix. }\end{array}$ & $\begin{array}{c}\text { Suspicious } \\
\text { intra-operative } \\
\text { findings }\end{array}$ & Type of tumour \\
\hline 1 & 65 & F & Yes & CT, ERCP & Yes & Yes & Moderately differentiated adenocarcinoma \\
2 & 69 & F & No & None & N/A & Yes & Moderately differentiated adenocarcinoma \\
3 & 78 & F & Yes & CT, MRCP & Yes & Yes & Moderately differentiated adenocarcinoma \\
4 & 59 & F & Yes & CT, MRCP & Yes & Yes & Poorly differentiated adenocarcinoma \\
5 & 90 & F & No & None & N/A & Yes & Non-specific adenocarcinoma \\
6 & 84 & F & Yes & CT & Yes & Yes & Moderately differentiated adenocarcinoma \\
7 & 83 & F & Yes & CT & Yes & Yes & Poorly differentiated adenocarcinoma \\
\hline
\end{tabular}

Table 3. Radiological Investigations and surgeon's findings with histological correlation. Adapted from De Zoysa M. I. M. et al. [5].

\begin{tabular}{|c|c|c|c|}
\hline \multirow{2}{*}{ Surgeon's and investigation findings } & \multicolumn{2}{|c|}{ Histopathology correlation } & \multirow{2}{*}{ Total } \\
\hline & Carcinoma & Benign & \\
\hline Normal investigations and normal surgeon's findings & 0 & 38 & $38 *$ \\
\hline Abnormal investigations and normal surgeon's findings & 0 & 3 & 3 \\
\hline Abnormal investigations and abnormal surgeon's findings. & 5 & 5 & 10 \\
\hline Normal investigations and abnormal surgeon's findings & 2 & 29 & 31 \\
\hline $\mathrm{P}$ value $=\mathbf{0 . 0 1 3} * *($ Fisher's exact testing $)$ & & & \\
\hline
\end{tabular}

*Denotes the number of gallbladder that would not have to be sent for histology; **Denotes statistical significance $\mathrm{P}<0.05$.

needs no further treatment apart from chole- cystectomy [4]. Wakai et al. in their study confirmed that most $\mathrm{pT}_{1 \mathrm{~b}}$ gallbladder carcinomas spread only locally and additional radical resection is not necessary when the depth of invasion of the gallbladder carcinoma is limited to the muscle layer after simple cholecystectomy [14] This argument is further corroborated from similar studies that presented evidence that the unsuspected cancers (either pre-operatively or intra-operatively) were not clinically significant in that knowledge of their presence did not alter subsequent management: patients had either complete resection of $\mathrm{pT}_{1}$ tumours or were unfit for adjuvant therapy/liver resection [15]. Later stages of carcinoma would present with macroscopic abnormalities and as such would be picked up in the criteria postulated in this study [8]. As such the main concern which would be to miss a very early staged carcinoma of the gallbladder has been addressed. This call for revised guidelines in sending all gallbladder samples to histology following cholecystectomy is echoed in other surgical departments in which they argue that the rarity of incidental histology findings relevant to patient management, especially in the absence of macro-scopic abnormalities, suggests that routine histological examination of certain specimens may be omitted [16].

Analysing a gallbladder specimen costs the NHS
$£ 10.40$ [17]. This works out at a cost of $£ 28038.40$ for the 2696 gallbladder specimens analysed in the Lancashire Teaching Hospitals Trust over the 5 year period. Taking into consideration that $46.3 \%$ of the gallbladders in our second study (Table 3) did not need to be sent to histology, this equates to 1248.2 patients. Therefore implementing the selective histology process mentioned the Trust would save itself approximately $£ 12,981$ over the 5 years, a saving of approximately $£ 2596$ each year. Coupled to that is the cost of employing someone to analyse the specimen. Consultant Histopathologist in the NHS gets paid approximately $£ 74,504-£ 100,446$ [18]. With the 48 hour working time directive that equates to $£ 29.85$ $£ 40.25$ an hour. In the approximate time of $15 \mathrm{mins}$ it takes to analyze a specimen [17] equates to approximately $£ 7.46-£ 10.06$ in wages. This works out as a saving of $£ 9311$ to $£ 12,554$ in hospital pay and a saving of approximately 312 hours (approximately 62 hours a year). In total there are potential savings of between $£ 22,292$ and $£ 25,535$ over the 5 year period. There are also significant time and pay savings when considering the time spent filling out specimen forms by surgical staff and the time it takes to transfer the gallbladder specimens. Advancing age should also play a part in stratifying risk and as such should also play a part in determining whether a gallbladder should be sent to histology. Given that 738 
gallbladders $(27 \%)$ were below 40 years of age, well below the youngest patient with carcinoma of the gallbladder at 59 years, this could also make way for further reductions. It is a well known fact that carcinoma of the gallbladder is more prevalent in females than in males [19]. It is also well known that certain ethnic groups are more susceptible to developing carcinoma of the gallbladder [20]. Combining all known risk factors as well as the data from radiological investigations, intra-operative macroscopic findings and lastly considering the clinical picture of the patient could further increase the accuracy of the selective criteria we have suggested in our study therefore saving more time and money.

Although the financial savings are apparently small in comparison to the billions that are spent each year in the NHS, the main saving is in time. Sub-specialization, increased clinical demand, more detailed analysis of specimens and minimum standards of reporting on difficult cases are frequent concerns among the pathology speciality in an environment in which time counts [3].

Similar studies reinforce the data from this study. Mittal R. et al. study found that gallbladder carcinoma was associated with macroscopic abnormalities in all the cases that they reviewed. As such they argue that there should be a restriction to sending away only those gallbladders to those that elicit macroscopic abnormalities [8]. Preliminary results from De Zoysa et al. [5] once again reinforce the idea of a more selective approach.

This study is more relevant today than ever before. With increased demand from the NHS, from financial burden's and subsequent layoffs as a consequence of the UK's debt there is increased pressure to provide a costeffective and efficient health care system. Implementing a more selective approach to histology during cholecystectomy can save valuable time and money. Coupled to this is a healthcare that is at the very tip of the evidence base but always remembering not to sacrifice patient safety in the face of financial gain.

This study could have been expanded to applying the selective criteria to all 2696 patients had there not been time constraints in order to increase the significance of the data. As such further studies are needed to corroborate such selective approaches either by way of retrospective analysis' or trials. The onus is on The Royal College of Pathologists (RCPath) to address the concerns from similar studies such as this one in order to achieve the goals they themselves set out to tackle in their published article "A recovery plan for histopathologists" in 2001 [21].

\section{Conclusion}

Over the last 5 years there have been 2696 cholecystectomies performed in the Lancashire Teaching Hospital
Trust. Of these there were 7 carcinoma's of the gallbladder, all of which could have been identified as being at high risk before the cholecystectomy based on criteria set out in this study. Using radiological investigations preoperatively and intra-operative macroscopic findings during the cholecystectomy this study suggests that there is a feasible and effective approach to reducing the number of gallbladders sent to histology therefore allowing for increased cost-effectiveness and time savings. Most importantly, patient safety is not compromised. Combined with well known risk factors such as increasing age, female sex, ethnicity and working on a when-in-doubt policy whereby if there are any suspicions whether small or large, all gallbladders should be sent for histology, clinicians will be able to put into action a more evidencebased approach to sending specimens to pathology.

\section{Acknowledgements}

I would also like to thank Andy Campbell for his help in obtaining the pathology reports and Deborah Fowler in her assistance in obtaining the patient medical records.

\section{REFERENCES}

[1] C. M. Lam, A. W. Yuen, A. C. Wai, R. M. Leung, A. Y. Lee, K. K. Ng, et al., "Gallbladder Cancer Presenting with Acute Cholecystitis. A Population-Based Study," Surgical Endoscopy, Vol. 19, No. 5, 2005, pp. 697-701. doi:10.1007/s00464-004-9116-2

[2] P. Cubertafond, M. Mathonnet, A. Gainant and B. Launois, "Radical Surgery for Gallbladder Cancer. Results of the French Surgical Society Survey," Hepato-Gastroenterology, Vol. 46, No. 27, 1999, pp. 1567-1571.

[3] N. A. Collier and L. H. Blumgart, "Tumours of the Gallbladder," In: L. H. Blumgart, Ed., Surgery of the Liver and Biliary Tract, 2nd Edition, Churchill Livingstone, New York, 1994, pp. 55-66.

[4] E. Yildirim, O. Celen, K. Gulben and U. Berberoglu, "The Surgical Management of Incidental Gallbladder Carcinoma," European Journal of Surgical Oncology, Vol. 31 , No. 1,2005 , pp. 45-52. doi:10.1016/j.ejso.2004.09.006

[5] M. I. M. De Zoysa, S. K. L. A. De Silva and A. Illiperuma, "Is Routine Histological Examination of the Gallbladder Specimens Justifiable?" Ceylon Medical Journal, Vol. 55, No. 1, 2010, pp. 3-16. doi:10.4038/cmj.v55i1.1702

[6] H. Gapaul, D. A. O'Reilly, J. Evans and D. Monk, "Is Routine Histology Part of 'a Perfect Cholecystectomy'?" British Journal of Surgery, Vol. 94, 2007, pp. 57-58.

[7] S. Mahmud, B. Darmas, A. Abbass, S. Paravastu, C. V. Rao and A. L. Baker, "Is There a Justification for Routine Histological Examination of the straightforward Cholecystectomy Specimens," British Journal of Surgery, Vol. 93, 2006, p. 117.

[8] R. Mittal, M. R. Jesudason and S. Nayak, "Selective 
Histopathology in Cholecystectomy for Gallstone Disease," Indian Journal of Gastroenterology, Vol. 29, No. 5, 2010, pp. 26-30. doi:10.1007/s12664-010-0056-6

[9] J. M. Piehler and R. W. Crichlow, "Primary Carcinoma of the Gallbladder," Surgery Gynecology \& Obstetrics, Vol. 147, 1978, pp. 929-942.

[10] J. Y. Jang, S. W. Kim, S. E. Lee, D. W. Hwang, E. J. Kim, J. Y. Lee, S. J. Kim and Y. T. Kim, "Differential Diagnosis and Staging Accuracies in High Resolution Ultrasonography, Endoscopic Ultrasonography and Multidetector Computed Tomography for Gallbladder Polyploid Lesions and Gallbladder Cancer," Annals of Surgery, Vol. 250 No. 6, 2009, pp. 943-949

[11] A. D. Levy, L. A. Murakata and C. A. Rohrmann, "Gallbladder Carcinoma: Radiological-Pathological Correlation," Radiographics, Vol. 21, No. 2, 2001, pp. 295-314.

[12] S. P. Shih, R. D. Schulick, J. L. Cameron, K. D. Lillemoe, H. A. Pitt, M. A. Choti, K. A. Campbell, C. J. Yeo and M. A. Talamini, "Gallbladder Cancer: The Role of Laparoscopic and Radical Resection," Annals of Surgery, Vol. 250, No. 3, 2009, p. 495.

[13] D. Morris-Stuff, J. Roche, M. E. Foster and M. H. Lewis, "The Incidence of Incidental Gallbladder Carcinoma Has Not Increased in the Laparoscopic Cholecystectomy Era," British Journal of Surgery, Vol. 95, Suppl. 7, 2008, p. 16.

[14] T. Wakai, Y. Shirai, N. Yokoyama, S. Nagakura, H. Watanabe and K. Hatakeyama, "Early Gallbladder Carcinoma
Does Not Warrant Radiacal Resection," British Journal of Surgery, Vol. 88, No. 5, 2001, pp. 675-678. doi:10.1046/j.1365-2168.2001.01749.x

[15] D. Roy, S. K. C. Toh, S. S. Somers, A. M. Walters and S. A. Sadek, "Value of Histology in a High-Volume Cholecystectomy Unit," British Journal of Surgery, Vol. 92, Suppl. 1, 2005, pp. 56-63.

[16] L. E. Matthyssens, M. Ziol, C. Barrat and G. G. Champault, "Routine Surgical Pathology in General Surgery," British Journal of Surgery, Vol. 93, 2006, pp. 362-368. doi:10.1002/bjs.5268

[17] F. P. Dix, I. A. Bruce, A. Krypcyzk and S. Ravi, "A Selective Approach to Histopathology of the Gallbladder Is Justifiable," Journal of the Royal Colleges of Edinburgh and Ireland, 2003, pp. 233-323.

[18] Reference for Income for a Histopathologist in the UK http://www.jobs.nhs.uk

[19] M. S. Khuroo, R. Mahajan, S. A. Zargar, G. Javid and S. Sapru, "Prevalence of Biliary Tract Disease in India: A Sonographic Study in Adult Population in Kashmir," GUT, Vol. 30, No. 2, 1989, pp. 201-205.

[20] G. M. Scarnecki, I. G. Karol and H. Khalil, "Gallbladder Carcinoma," 2011. www.medscape.com

[21] J. C. E. Underwood, "A Recovery Plan for Histopathology," Royal College Pathology Bulletin, Vol. 113, 2001, pp. 12-14. 